\title{
The effect of like-charge attraction on aerosol growth in the atmosphere of Titan
}

\author{
Eric B. Lindgren ${ }^{\mathrm{a}}$, Benjamin Stamm ${ }^{\mathrm{b}, \mathrm{c}}$, Ho-Kei Chan ${ }^{\mathrm{a}, 1}$, Yvon Maday ${ }^{\mathrm{d}, \mathrm{e}, \mathrm{f}, \mathrm{g}}$, \\ Anthony J. Stace ${ }^{\mathrm{a}}$, Elena Besley ${ }^{\mathrm{a}, *}$ \\ ${ }^{a}$ Department of Physical and Theoretical Chemistry, School of Chemistry, University of \\ Nottingham, University Park, Nottingham NG7 2RD, United Kingdom \\ ${ }^{b}$ Center for Computational Engineering, Mathematics Department, RWTH Aachen \\ University, Schinkelstr. 2, 52062 Aachen, Germany \\ ${ }^{c}$ Computational Biomedicine, Institute for Advanced Simulation IAS-5 and Institute of \\ Neuroscience and Medicine INM-9, Forschungszentrum Jülich, Germany \\ ${ }^{d}$ Sorbonne Universités, UPMC Univ Paris 06, UMR 7598, Laboratoire Jacques-Louis Lions, \\ F-75005, Paris, France \\ ${ }^{e}$ CNRS, UMR 7598, Laboratoire Jacques-Louis Lions, F-75005, Paris, France \\ ${ }^{f}$ Institut Universitaire de France \\ ${ }^{g}$ Brown Univ, Division of Applied Maths, Providence, RI, USA
}

\begin{abstract}
The formation of aerosols in the atmosphere of Titan is based extensively on ion-neutral chemistry and physical condensation processes. Herein it is shown that the formation of aerosols may also occur through an alternative pathway that involves the physical aggregation of negatively charged particles, which are known to be abundant in the satellite's atmosphere. It is shown that, given the right circumstances, like-charged particles with a dielectric constant characteristic of nitrated hydrocarbons have sufficient kinetic energy to overcome any repulsive electrostatic barrier that separates them and can subsequently experience an attractive interaction at very short separation. Aerosol growth can then unfold through a charge scavenging process, whereby nitrated aggregates preferentially grow by assimilating smaller like-charged particles. Since hydrocarbon aerosols have much lower dielectric constants, it is shown that a similar mechanism involving hydrocarbon particles will not be as efficient. As a
\end{abstract}

\footnotetext{
* Corresponding author

Email address: elena.besley@nottingham.ac.uk (Elena Besley)

${ }^{1}$ Current address: Shenzhen Graduate School, The Harbin Institute of Technology, Shenzhen, China.
} 
consequence of this proposed growth mechanism, it is suggested that the lower atmosphere of Titan will be enriched in nitrogen-containing aerosols.

Keywords: Titan, Titan, atmosphere

\section{Introduction}

Since the discovery of Titan by the Dutch astronomer Christiaan Huygens in 1655, Saturn's largest moon has been subject of considerable interest and curiosity. Remarkably, Titan is the only satellite in the Solar System known

5 to display a dense atmosphere [1, harbouring several organic compounds and particular conditions that led some authors to consider the presence of different life forms [2, 3, 4, 5. Over the past few decades, a significant amount of new information about Titan has been acquired through the Voyager program and, latterly, with the Cassini-Huygens mission. While Voyager represented a breakthrough in exploration of the Solar System and, particularly, Titan, it was only with arrival of the Cassini-Huygens spacecraft in 2004 at Saturn and its moons that Titan's atmosphere and surface could be studied in greater detail, through in situ measurements made by the lander Huygens and the Cassini orbiter.

The atmosphere of Titan is known to stage a series of chemical and physical 15 processes that are ultimately responsible for the formation of a characteristic orange haze. Composed primarily of nitrogen $\left(\mathrm{N}_{2}\right)$ and methane $\left(\mathrm{CH}_{4}\right)$ [1, 6, 7], Titan's atmosphere is constantly bombarded by energy sources such as solar ultraviolet radiation and energetic particles from Saturn's magnetosphere. This irradiation triggers the dissociation and ionization of the simple primordial molecules and subsequently leads, through a series of chemical and physical processes, to the formation of charged aerosol particles with an average mass of $500 \mathrm{Da}$ [8] at altitudes between 950 and $1150 \mathrm{~km}$ (upper atmosphere) [6, 7, 9, 10, 11. With decreasing altitude, these particles grow spherically until they reach the detached haze layer at $520 \mathrm{~km}$ in Titan's mesosphere, from where they 25 start to form fractal aggregates [7, 12, 13, 14].

Several studies have already shown the crucial role of ion-neutral chemistry 
and physical aggregation in the production of aerosols in Titan's atmosphere [7, 8, 9, 12, 13, 14, 15, 16, 17, 18, 19, 20, 21. In this paper, we present a theoretical investigation of a particular aspect of aerosol growth that has not previously been considered. New calculations have been undertaken for the case of likecharged particle interactions in vacuum, which, under certain circumstances, can be counterintuitively attractive. Interactions between neutral and charged particles as well as between oppositely charged particles are not considered since these are already taken to be attractive under similar conditions. The calculations examine how differences in the dielectric constants of particles could lead to a preferential concentration of nitrogen-containing aerosols in the lower atmosphere of Titan.

Depending on the assumed mass density, particles produced in the upper atmosphere of Titan have an estimated radius of either $0.6 \mathrm{~nm}$ or $6 \mathrm{~nm}$ if

40 1 elementary charge per particle and densities of $1000 \mathrm{~kg} \cdot \mathrm{m}^{-3}$ [ 8 ] or $1 \mathrm{~kg}$ $\cdot \mathrm{m}^{-3}$ [22, 23], respectively, are considered. Regardless of their initial size, these particles grow as they sink towards lower altitudes eventually forming large particles with radii of $40-50 \mathrm{~nm}$, which have been identified as being present in the detached haze layer [12, 13, 24, 25]. Since the amount of charge on 45 the embryonic particles cannot be independently measured, actual sizes could be larger than those estimated above [6, 9]. For example, a particle bearing 5 elementary charges would have a radius 1.7 greater than the same particle carrying just 1 charge. To take into account this degree of uncertainty, the calculations presented here consider charge per particle values that range from 1 to $5 \mathrm{e}$.

\section{Origin of like-charge attraction}

In Titan's atmosphere it is generally assumed that like-charged particles will repel one another and so any such interactions will make no contribution to the growth of aerosols [8]. Whilst this concept is generally true, if the particles are sufficiently polarizable, attraction can occur at small separations. Bichout- 
skaia et al [26] showed that for two interacting dielectric particles in vacuum, the electrostatic force can be expressed as the sum of two terms - a repulsive term described by Coulomb's law and an attractive polarization term. If the particles are non-polarizable, namely if their dielectric constant, $k_{i}(i=1,2)$, is close to unity, the attractive term tends to zero and the total force is described by Coulomb's law. Conversely, if the particles have dielectric constants that are much greater than unity then the attractive polarization term gains in magnitude and, under certain circumstances, can even overcome the repulsion. As presented, the formalism [26] is able to treat a broad range of dielectric materials, and is able to differentiate between particles with very different polarizabilities, ranging from oils $(k \approx 2)$ through to water $(k \approx 80)$, and on to particles of metallic character $(k \geq 1000)$.
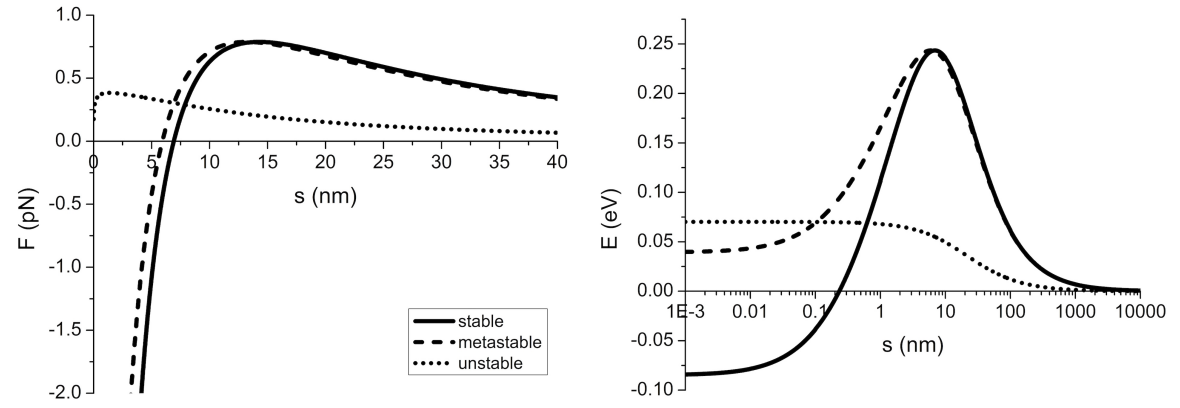

Figure 1: (a) Electrostatic force $(\mathrm{pN})$ and (b) potential energy (eV) curves for cases of stable (solid line; $a_{2}=6 \mathrm{~nm}, q_{2}=5 \mathrm{e}$ ), metastable (dashed line; $a_{2}=7 \mathrm{~nm}, q_{2}=5 \mathrm{e}$ ) and unstable (dotted line; $a_{2}=8 \mathrm{~nm}, q_{2}=1 \mathrm{e}$ ) interactions. The dielectric constant of both particles, and the radius and charge of particle 1 are maintained fixed at $k_{1}=k_{2}=20, a_{1}=10 \mathrm{~nm}$ and $q_{1}=1 \mathrm{e}$, respectively. Surface-to-surface separation $(s)$ and radii are given in nanometers, and charges in elementary unit. Note that the scale of the $x$-axis is different on each plot.

Figure 1 shows how the electrostatic force (a) and potential energy (b) varies as a function of particle separation for three different cases of interacting likecharged dielectric particles. Each particle has been assigned a dielectric constant of $k_{i}=20$, since this value is comparable to that of many hydrocarbonnitrile compounds [27, and so should be representative of nitrile-rich aerosols. 
Charges, $q_{i}$, and radii, $a_{i}$, were also selected in order to represent emerging particles in the region between the upper atmosphere and the detached haze layer of Titan's atmosphere. The curve depicting an unstable interaction (dotted line) corresponds to a scenario where the particles repel one another at all values of interparticle separation, which means the potential energy increases continuously during a collision. The metastable (dashed line) and stable (solid line) cases are similar in that a repulsive potential energy barrier occurs before they achieve close separation; however, once over the barrier, the energy decreases rapidly as the particles approach touching point. The reason for the observed decrease in potential energy is the onset of an overall polarization induced attractive interaction at sufficiently close interparticle separation. Accordingly, it can be proposed that particles interacting under a stable or even metastable electrostatic regime may provide an important contribution to the growth of aerosol particles and production of the 40-50 $\mathrm{nm}$ radius aerosols found in the detached haze layer. It is especially significant when it is considered that charged particles, particularly those carrying a negative charge, are present in high abundance in Titan's atmosphere [8]. The circumstances where such stabilising interactions occur and how the interacting particles are able to overcome the repulsive Coulomb barrier are discussed below. Details of the calculations can be found in Theory section, where a description is given of the steps taken to achieve a rapid convergence of the electrostatic multipole terms that arise in the formalism describing charge particle interactions. It has recently been shown that a failure to include sufficient terms can lead to a very considerable underestimate in the magnitude of polarization interactions [28].

\section{Discussion}

Table $1(\mathrm{a}-\mathrm{e})$ shows values of potential energy barriers $(\mathrm{eV})$ calculated for different cases where two like-charged particles interact. These examples have been chosen to represent the process of particle growth that may occur within Titan's upper atmosphere, leading to the production of aerosol monomers in the 
detached haze layer. In each sub-table, the radius $a_{2}$ and charge $q_{2}$ of sphere 2 have been allowed to vary from 5 to $50 \mathrm{~nm}$ and 1 to 5 e, respectively, whilst the equivalent parameters $a_{1}$ and $q_{1}$ for sphere 1 have remained fixed.

As can be seen from Table 1, examples of unstable interactions occur at or near the point where the interacting particles have identical characteristics, i.e. $a_{1}=a_{2}$ and $q_{1}=q_{2}$, and in general they extend across points where $a_{2} / a_{1}$ and $q_{2} / q_{1}$ increase concurrently. Cases of stable interaction occur when the particles have sufficiently dissimilar sizes, and are more generally favoured when the smaller of the two particles carries more charge. Metastable interactions fall between these two scenarios. The magnitude of the energy barrier varies inversely with particle radii and increases with the amount of charge. Broadly speaking, as interacting particles increase in size, the free charge on one becomes, on average, more distant from the free charge on the second particle, which then contributes to a decrease in Coulomb repulsion and, consequently, a decrease in the energy barrier. In contrast, for a fixed particle size, an increase in the amount of free charge on one or both particles means that more charge occupies a fixed area, which then contributes to an increase in Coulomb repulsion and, consequently, an increase in the magnitude of the energy barrier.

Figure 2 a shows a Maxwell-Boltzmann distribution of the kinetic energy of particles calculated for the upper and lower average temperatures, $112 \mathrm{~K}$ and 175 K [29], as identified for Titan's thermosphere. The calculations presented in Table 1 suggest that at the beginning of the aerosol growth process, when particles are particularly small, the Coulomb repulsion barrier, generally greater than $k T$, is high enough to reduce the coagulation rates between like-charged particles. Hence growth is restricted to oppositely charged interactions, and notably the absorption of positive ions by the large negatively charged particles [8], or even neutral-charged particle interactions. However, as particle growth continues the charge density will drop and the corresponding Coulomb barrier will decrease in magnitude and will eventually become comparable to or even smaller than $k T$. In which case, a colliding pair of like-charged particles could interact under stable or metastable conditions and contribute to the growth 
Table 1: Calculated energy barriers (eV) between two interacting like-charged, dielectric particles, for a range of radii ( $\mathrm{nm}$ ) and charge (e). White, light and dark gray shades denote stable, metastable, and unstable interactions, respectively. The charge $q_{1}$ and $q_{2}$ can be regarded as either positive or negative.

\begin{tabular}{|c|c|c|c|c|c|c|c|c|c|c|}
\hline 5 & 0.49 & 0.43 & 0.33 & 0.25 & 0.20 & 0.17 & 0.14 & 0.13 & 0.11 & 0.10 \\
\hline 4 & 0.41 & 0.35 & 0.25 & 0.19 & 0.16 & 0.13 & 0.11 & 0.097 & 0.086 & 0.078 \\
\hline 3 & 0.33 & 0.26 & 0.18 & 0.14 & 0.11 & 0.092 & 0.079 & 0.069 & 0.062 & 0.055 \\
\hline 2 & 0.24 & 0.17 & 0.11 & 0.086 & 0.069 & 0.057 & 0.049 & 0.043 & 0.038 & 0.034 \\
\hline 1 & 0.13 & 0.074 & 0.049 & 0.037 & 0.030 & 0.025 & 0.021 & 0.019 & 0.016 & 0.015 \\
\hline$a_{2}$ & 5 & 10 & 15 & 20 & 25 & 30 & 35 & 40 & 45 & 50 \\
\hline
\end{tabular}

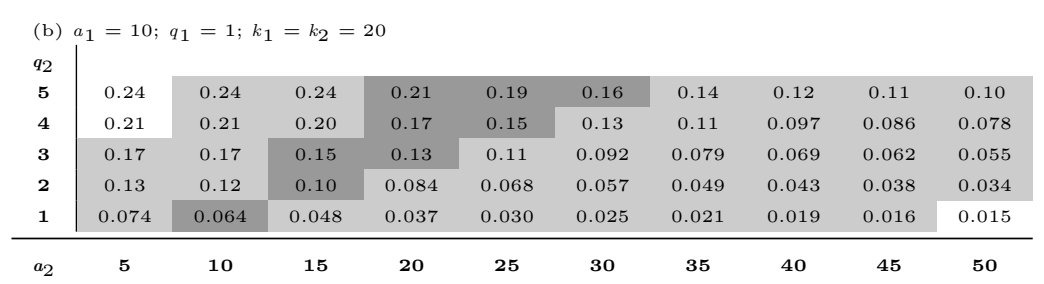

\begin{tabular}{|c|c|c|c|c|c|c|c|c|c|c|}
\hline 5 & 0.12 & 0.12 & 0.12 & 0.12 & 0.12 & 0.12 & 0.11 & 0.11 & 0.10 & 0.094 \\
\hline 4 & 0.10 & 0.10 & 0.10 & 0.10 & 0.10 & 0.098 & 0.093 & 0.087 & 0.080 & 0.074 \\
\hline 3 & 0.084 & 0.084 & 0.084 & 0.083 & 0.081 & 0.076 & 0.071 & 0.065 & 0.059 & 0.054 \\
\hline 2 & 0.063 & 0.063 & 0.062 & 0.061 & 0.057 & 0.052 & 0.047 & 0.042 & 0.038 & 0.034 \\
\hline 1 & 0.037 & 0.037 & 0.036 & 0.032 & 0.028 & 0.024 & 0.021 & 0.018 & 0.016 & 0.015 \\
\hline$a_{2}$ & 5 & 10 & 15 & 20 & 25 & 30 & 35 & 40 & 45 & 50 \\
\hline
\end{tabular}

\begin{tabular}{|c|c|c|c|c|c|c|c|c|c|c|}
\hline 5 & 0.081 & 0.081 & 0.081 & 0.081 & 0.081 & 0.081 & 0.081 & 0.080 & 0.079 & 0.077 \\
\hline 4 & 0.069 & 0.069 & 0.069 & 0.069 & 0.069 & 0.069 & 0.068 & 0.067 & 0.065 & 0.063 \\
\hline 3 & 0.056 & 0.056 & 0.056 & 0.056 & 0.056 & 0.056 & 0.055 & 0.053 & 0.051 & 0.048 \\
\hline 2 & 0.042 & 0.042 & 0.042 & 0.042 & 0.041 & 0.040 & 0.039 & 0.037 & 0.034 & 0.032 \\
\hline 1 & 0.025 & 0.025 & 0.025 & 0.024 & 0.023 & 0.021 & 0.020 & 0.018 & 0.016 & 0.015 \\
\hline & 5 & 10 & 15 & 20 & 25 & 30 & 35 & 40 & 45 & 50 \\
\hline
\end{tabular}

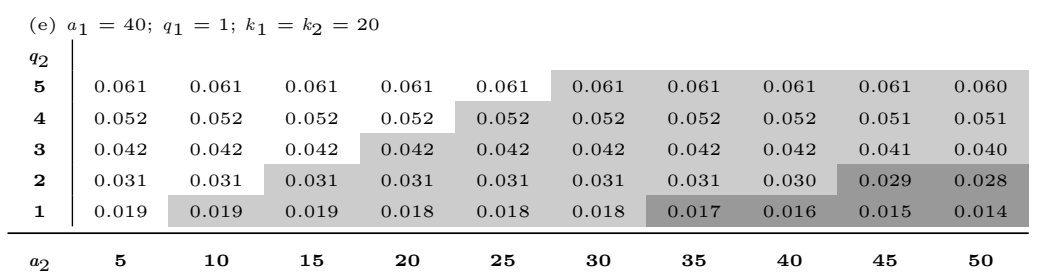


(a)

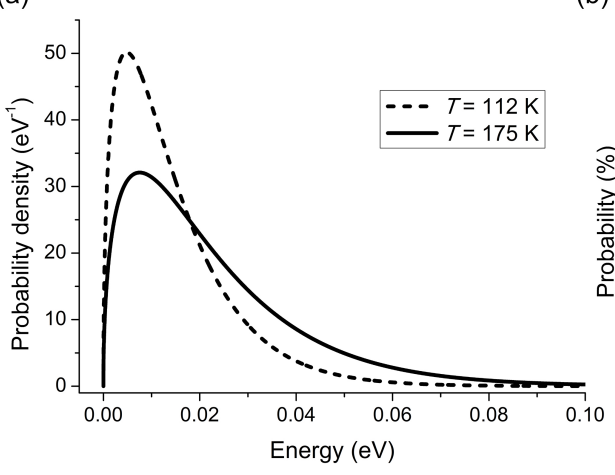

(b)

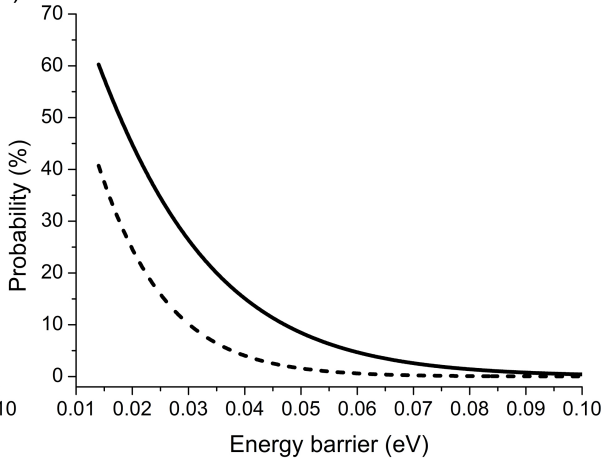

Figure 2: (a) Maxwell-Boltzmann distribution of the particle kinect energy at the upper and lower Titan's thermosphere average temperatures. (b) Probability (\%) that particles, with a thermal energy of $k T$, will overcome the barrier as function of the latter's magnitude, evaluated for the two extreme temperatures. In the second plot, any point after the maximum value for the $\mathrm{x}$-axis has a corresponding probability that is always smaller than $0.5 \%$.

process. For example, taking the case shown in Table 1(c) where $a_{1}=20 \mathrm{~nm}$, $a_{2}=10 \mathrm{~nm}$ and $q_{1}=q_{2}=1 \mathrm{e}$, the interaction is predicted to be metastable with a barrier height of $0.037 \mathrm{eV}$. According to the distribution of kinetic energies shown in Figure $2 \mathrm{~b}$, there is, at the above temperatures, an $8-18 \%$ probability that the particles will have sufficient energy to overcome the barrier. Any barrier higher than $0.1 \mathrm{eV}$ is unlikely to be overcome.

In cases that involve low Coulomb barriers it can be considered that likecharged interactions, especially those between the more abundant negatively charged particles, can contribute to growth via a scavenging mechanism, similar to that identified for the formation of clouds on Earth [30, 31, 32. In that case, sufficiently large aerosol droplets will grow by assimilating smaller particles even when they are like-charged. This mechanism should then be regarded as an important component of the whole aerosol growth process, along with the usually considered interactions between oppositely charged particles and neutral-charged particle collisions. Table 1 also suggests that an increase in the Coulomb repulsion barrier with the amount of charge residing on particles should prevent large departures from neutrality with respect to the growing ag- 
gregates. Therefore, the absorption of positive ions by large negatively charged particles and a charge scavenging mechanism are very likely to operate synergistically. The presence of positively charged particles would act to keep a low net charge on growing particles, whilst a charge scavenging mechanism would be highly favourable due to the higher abundance of negatively charged particles. 55 It is worth mentioning that this mechanism operates irrespective of the sign of particle's charge. That is, if there is an initial bias towards, for instance, the production negative ions, as suggested by experimental evidence to be the case for Titan [8, 33, 34, 35], then the mechanism will act to enhance the growth of such particles until possibly reaching an upper limit to the amount of charge each growing particle can carry when Coulomb repulsion becomes the dominant interaction. Similarly, if the initial bias is towards the production of positive ions then the mechanism would operate identically, since the magnitude of electrostatic interactions is independent of sign of charge (vide Coulomb's law). As already noted elsewhere 22], the preferential formation of negatively charged particles seems understandable considering the electron affinity of the molecules that compose Titan's upper atmosphere.

Table 2 shows the consequences of like-charged particles interacting under circumstances identical to those given in Table 1 (b), but with a dielectric constant of $k_{1}=k_{2}=3$. Such a value would be appropriate for many hydrocarbon and polycyclic aromatic hydrocarbon (PAH) compounds [27]. As can be seen from Table 2, there are no interactions where the outcome of a collision would be a larger, stable particle. There are a few metastable interactions, but the majority of collisions would take place on a repulsive energy surface and lead to an immediate separation of particles. For the most part, energy barriers are also higher than those calculated when $k_{1}=k_{2}=20$. Consequently, it could be inferred that an enrichment in nitrogen content of Titan's atmospheric aerosols might be favoured as a consequence of a charge scavenging mechanism operated by the abundant large negatively charged particles. Critical to the effectiveness of this enrichment mechanism is the large difference in dielectric constant that exists between hydrocarbon-based particles and those where the 
constituent molecules predominantly include nitrogen atoms.

Table 2: Energy barrier $(\mathrm{eV})$ for the case of two interacting dielectric particles as depicted in Table 1.(b), but with a lower dielectric constant of $k_{1}=k_{2}=3$.

\begin{tabular}{c|cccccccccc}
$q_{2}$ & & & & & & & & & & \\
$\mathbf{5}$ & 0.30 & 0.30 & 0.26 & 0.23 & 0.20 & 0.17 & 0.15 & 0.13 & 0.12 & 0.11 \\
$\mathbf{4}$ & 0.25 & 0.25 & 0.21 & 0.18 & 0.16 & 0.14 & 0.12 & 0.11 & 0.095 & 0.085 \\
$\mathbf{3}$ & 0.20 & 0.19 & 0.16 & 0.14 & 0.12 & 0.10 & 0.087 & 0.077 & 0.069 & 0.062 \\
$\mathbf{2}$ & 0.15 & 0.13 & 0.11 & 0.090 & 0.075 & 0.064 & 0.055 & 0.049 & 0.043 & 0.039 \\
$\mathbf{1}$ & 0.085 & 0.068 & 0.053 & 0.042 & 0.034 & 0.029 & 0.025 & 0.022 & 0.019 & 0.017 \\
\hline $\boldsymbol{a}_{\mathbf{2}}$ & $\mathbf{5}$ & $\mathbf{1 0}$ & $\mathbf{1 5}$ & $\mathbf{2 0}$ & $\mathbf{2 5}$ & $\mathbf{3 0}$ & $\mathbf{3 5}$ & $\mathbf{4 0}$ & $\mathbf{4 5}$ & $\mathbf{5 0}$
\end{tabular}

A lack of nitrogen atoms in the constituent molecules of a particle, for example, a droplet that is mostly composed of methane or ethane, would lead to a considerable reduction in dielectric constant, which in turn, would make attractive or stabilising like-charge interactions much less likely. Pure hydrocarbons generally have low dielectric constants due to their non-polar character. Methane $\left(\mathrm{CH}_{4}\right)$ and ethane $\left(\mathrm{C}_{2} \mathrm{H}_{6}\right)$, compounds that are presented at an early stage of aerosol formation in Titan's atmosphere, have dielectric constant values of just 1.6761 and 1.9356, respectively [27]. Polar-substituted hydrocarbons naturally have higher dielectric constants, so that the incorporation of a functional group containing nitrogen, for example, usually increases the dielectric constant of the substituted hydrocarbon. Hydrogen cyanide (HCN), one of the main nitrated compounds found in Titan's atmosphere, has a particular high dielectric constant value of 114.9 [27]. Figure 3 shows estimated values (according to [36]) for the dielectric constant of a mixture consisting of methane/hydrogen cyanide, and ethane/hydrogen cyanide, both as function of the molar fraction of hydrogen cyanide $\left(\mathrm{x}_{\mathrm{HCN}}\right)$. As can be seen, a mixture that contains at least about 20 percent of molar fraction of $\mathrm{HCN}$ will have a dielectric constant of 20 or greater in both cases; stressing the assertion that the incorporation of nitrogen by the growing aerosol particle favours the scavenging mechanism.

As part of this growth process, it should be noted that all other factors which contribute towards the calculation of collision efficiencies, i.e. Reynolds number, terminal velocity, etc., will be identical for all particles with the same radius, 


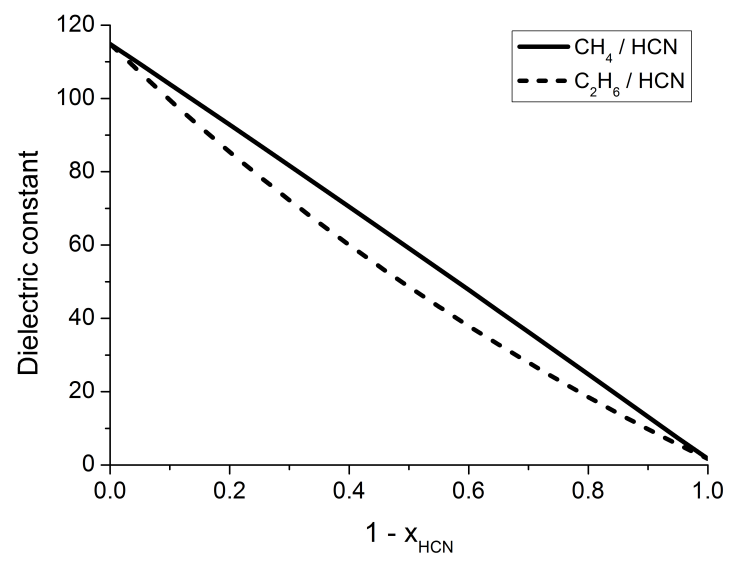

Figure 3: Plot of the dielectric constant as function of hydrogen cyanide molar fraction for methane/hydrogen cyanide (solid line) and ethane/hydrogen cyanide (dotted line) mixtures.

mass and charge, irrespective of composition [37. Hence, the only quantity that will differentiate collision efficiencies and, as a result, the degree of enrichment for each of the cases in Tables 1 and 2 is the dielectric constant.

\section{Theory}

A recently presented solution [26 to the problem of calculating electrostatic interactions between charged particles of dielectric materials has been used to determine electrostatic forces and potential energies. The model takes as input the charge $q_{i}$, radius $a_{i}$, and dielectric constant $k_{i}$ of each particle $(i=1,2)$, and their separation distance. The model assumes that polarization and free charge reside on the surfaces of the particles, and that the total surface charge density can be written as the sum of contributions of these two components, $\sigma_{i}=\sigma_{p, i}+$ $\sigma_{f, i}$. Figure 4 gives a geometric representation of the problem being addressed. The spherical geometry is in accordance with the spherical nature of the particles that form aggregates found above Titan's stratosphere. [7, 12, 13, 14. The free charge is assumed to be uniformly distributed; in contrast, the polarization charge on each particle is treated as a dynamic quantity that varies in response to the electric field created by charge residing on the second particle, which in 
turn can be found according to electrostatics [26].

The laws that govern the electric potential $\Phi$ associated with the problem of two interacting particles can be stated as follows:

$$
-\operatorname{div}(k \nabla \Phi)=4 \pi K \sigma_{f}
$$

which states that the normal component of the electric displacement field is discontinuous due to the presence of free charge $\sigma_{f}$ on the surface of each particle $\Gamma_{i}, i=1,2$ :

$$
\sigma_{f}(x)=\left\{\begin{aligned}
\sigma_{f, i} & \text { if } x \in \Gamma_{i} \\
0 & \text { otherwise. }
\end{aligned}\right.
$$

Note that the function $k$ takes the value of 1 on the outside of the two spheres and the value of $k_{i}$ inside sphere $i$. This implies that $\Phi$ is a continuous function that is harmonic both on the inside and the outside of the particles. This potential can thus be represented by a global single layer potential

$$
\Phi=\tilde{\mathcal{S}}_{1}\left(\sigma_{1}\right)+\tilde{\mathcal{S}}_{2}\left(\sigma_{2}\right)
$$

where $\sigma_{i}$ is the total surface charge resulting from the sum of the free $\sigma_{f, i}$ and bound (polarization) charges $\sigma_{p, i}$ on each sphere. Since the particles are spheres, each $\sigma_{i}$ can be represented in terms of spherical harmonics on each sphere. In addition, the axial invariance of the problem allows cancellation of the dependency on azimuthal angle such that the following development holds

$$
\sigma_{i}(x)=\frac{1}{4 \pi K} \sum_{\ell=0}^{\infty} A_{i, \ell} \frac{2 \ell+1}{a_{i}^{\ell+2}} \mathcal{Y}_{\ell, 0}\left(\frac{x-x_{i}}{a_{i}}\right),
$$

the coordinates $x_{i}$ being the center of the $i$-th sphere.

Equation (1) 26], implies that

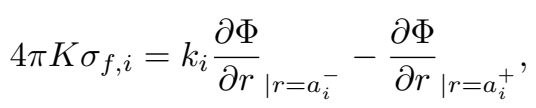




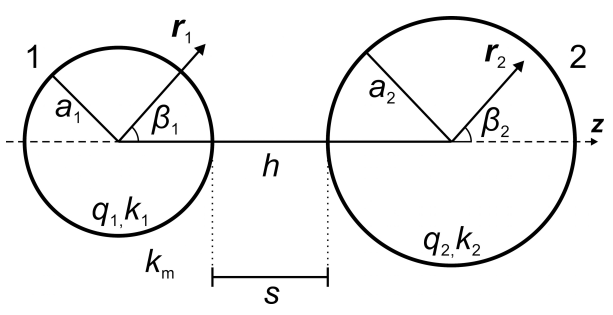

Figure 4: Geometric representation of the system formed by two interacting charged dielectric spheres. Charge, radius, dielectric constant, and the polar angle of each sphere $(i=1,2)$ are denoted as $q_{i}, a_{i}, k_{i}$, and $\beta_{i}$ respectively. The center-to-center separation is defined as $h$ and the corresponding surface-to-surface separation is given by $s=h-a_{1}-a_{2}$. The particles are assumed to be suspended in a vacuum, with dielectric constant of $k_{m}=1$.

The continuity of the normal derivative of $\tilde{\mathcal{S}}_{i}\left(\sigma_{i}\right)$ through $\Gamma_{j}$ for $j \neq i$ yields $\Gamma_{i}$ such that

$$
\forall \ell, \quad 4 \pi K a_{i} \sigma_{f, i} \delta_{\ell, 0}=\frac{A_{i, \ell}}{a_{i}^{\ell+1}}+\frac{\left(k_{i}-1\right) \ell}{\left(k_{i}+1\right) \ell+1} \sum_{m=0}^{\infty} A_{j, m} \frac{(\ell+m) !}{\ell ! m !} \frac{a_{i}^{\ell}}{h^{\ell+m+1}},
$$

with $j=\bmod (i, 2)+1$, see [26]. In order to symmetrize the system, the quantities $\tilde{A}_{i, m}=\frac{A_{i, m}}{a_{i}^{m+1}}$ can be introduced and the above infinite-dimensional system can

be rewritten as

$$
\forall \ell, \quad 4 \pi K a_{i} \sigma_{f, i} \delta_{\ell, 0}=\tilde{A}_{i, \ell}+\frac{a_{j}}{h} \frac{\left(k_{i}-1\right) \ell}{\left(k_{i}+1\right) \ell+1} \sum_{m=0}^{\infty} \tilde{A}_{j, m}\left(\tilde{d}_{i}\right)_{\ell m} .
$$

with

$$
\left(\tilde{d}_{i}\right)_{\ell m}=\frac{(\ell+m) !}{\ell ! m !}\left(\frac{a_{i}}{h}\right)^{\ell}\left(\frac{a_{j}}{h}\right)^{m} .
$$

The first immediate observation is that, for $\ell=0$, the following equation is obtained:

$$
\tilde{A}_{i, 0}=4 \pi K a_{i} \sigma_{f, i} .
$$


By substituting (8) into (7), and after some algebra the following infinite

$$
\forall \ell>0, \quad \frac{h}{a_{j}} \frac{\left(k_{i}+1\right) \ell+1}{\left(k_{i}-1\right) \ell} \widetilde{A}_{i, \ell}+\sum_{m=1}^{\infty}\left(\tilde{d}_{i}\right)_{\ell m} \widetilde{A}_{j, m}=-\left(\tilde{d}_{i}\right)_{\ell 0} \widetilde{A}_{j, 0},
$$

which is symmetric since $\left(\tilde{d}_{1}\right)_{\ell m}=\left(\tilde{d}_{2}\right)_{m \ell}$ and can be solved efficiently after truncation, as explained in the Appendix.

The electrostatic force is obtained from a generalization of Coulomb's law for point particles given by

$$
\mathbf{F}_{12}=K \int d q_{1}\left(\mathbf{r}_{1}\right) \int d q_{2}\left(\mathbf{r}_{2}\right) \frac{\mathbf{r}_{1}-\mathbf{r}_{2}}{\left|\mathbf{r}_{1}-\mathbf{r}_{2}\right|^{3}},
$$

where $\mathbf{r}_{1}$ and $\mathbf{r}_{2}$ are position vectors at the surface of particles 1 and 2 , respectively, $d q_{1}\left(\mathbf{r}_{1}\right)$ and $d q_{2}\left(\mathbf{r}_{2}\right)$ are the charge elements of each particle, and $K$ is the Coulomb's constant. The first integral takes into account the charge residing on particle 1 and the second integral is the potential generated by the charge residing on particle 2. The force, $\mathbf{F}_{12}$, is evaluated by the expansion in terms as they interact. The charge distribution, $\sigma_{i}$, on the surfaces of the particles is integrated, to give the following analytic expression for the electrostatic force

$$
\begin{aligned}
\mathbf{F}_{12}= & K \frac{q_{1} q_{2}}{h^{2}}-q_{1} \sum_{m=1}^{\infty} \sum_{l=0}^{\infty} A_{1, l} \frac{\left(k_{2}-1\right) m(m+1)}{\left(k_{2}+1\right) m+1} \\
& \times \frac{(l+m) !}{l ! m !} \frac{a_{2}^{2 m+1}}{h^{2 m+l+3}}-\frac{1}{K} \sum_{l=1}^{\infty} A_{1, l} A_{1, l+1} \frac{\left(k_{1}+1\right)(l+1)+1}{\left(k_{1}-1\right) a_{1}^{2 l+3}} .
\end{aligned}
$$

which can then be integrated numerically to give the potential energy.

\section{Conclusions}

Theory developed to account for electrostatic interactions between charged particles composed from dielectric materials has been applied to the growth of 
aerosols in the atmosphere of Titan. The calculations show that, under circumstances where there is an abundance of like-charged particles, the value of the dielectric constant for a particular material can have a significant effect on efficiency with which particles increase in size. For the case of Titan, it is proposed that this process, akin to electrostatic enrichment, could lead to the preferential growth of aerosols which contain an abundance of nitrated hydrocarbons. A similar mechanism could apply to other atmospheres where the constituent aerosols display a wide range of dielectric constants.

\section{Acknowledgments}

EBL is supported by a PhD scholarship from the Brazilian Government's Science Without Borders programme (CAPES: 0702/13-7). Part of this work (associated with YM and BS) has benefited from French state funding managed by CALSIMLAB and the ANR within the Investissements d'Avenir programme under reference ANR-11-IDEX-0004-02. EB acknowledges the financial support of an ERC Consolidator grant. The authors would like to acknowledge Prof. A. J. Coates for his critical reading of the manuscript.

\section{Appendix A: Numerical approximation}

The first step in this part is to discretize the infinite sum given by equation

That is, two vectors $\widetilde{A}_{1}^{N}$ and $\widetilde{A}_{2}^{N}$, each of dimension $N$ and with coefficients $\widetilde{A}_{i, \ell}^{N}$ are sought, satisfying the linear system

$$
\begin{aligned}
& C_{1} \widetilde{A}_{1}^{N}+D_{1} \widetilde{A}_{2}^{N}=b_{1} \\
& D_{2} \widetilde{A}_{1}^{N}+C_{2} \widetilde{A}_{2}^{N}=b_{2}
\end{aligned}
$$

of size $(2 N) \times(2 N)$ with a symmetric solution matrix. Here, the vectors $b_{i}$ and the matrices $C_{i}$ and $D_{i}$ are given by 


$$
\left(b_{i}\right)_{\ell}=-\tilde{d}_{i, \ell 0} \widetilde{A}_{j, 0}, \quad\left(C_{i}\right)_{\ell m}=\frac{h}{a_{j}} \frac{\left(k_{i}+1\right) \ell+1}{\left(k_{i}-1\right) \ell} \delta_{\ell m}, \quad\left(D_{i}\right)_{\ell m}=\left(\tilde{d}_{i}\right)_{\ell m},
$$

for all $\ell, m=1, \ldots, N$. From the solution to this system, as in (4), an approximation can be reconstructed

$$
\sigma_{i}^{N}=\frac{1}{4 \pi K a_{i}}\left[\tilde{A}_{i, 0} \mathcal{Y}_{0,0}+\sum_{\ell=1}^{N} \tilde{A}_{i, \ell}^{N}(2 \ell+1) \mathcal{Y}_{\ell, 0}\left(\frac{x-x_{i}}{a_{i}}\right)\right],
$$

to $\sigma_{i}, i=1,2$, that can be shown to converge rapidly. Recall that $\tilde{A}_{i, 0}$ is given by (8). This, in turn, provides an approximation to the electrostatic force (11). Since this matrix problem 12 is symmetric, solution by an iterative conjugate gradient method is an natural choice. In order to provide an illustration of the complexity, analysis on the convergence has been performed and is presented in the plots below. These graphs are all related to the same selection of parameters, namely: $k_{1}=k_{2}=20, a_{1}=5 \mathrm{~nm}, a_{2}=10 \mathrm{~nm}, q_{1}=q_{2}=1 \mathrm{e}$, and $s=0.01$ $\mathrm{nm}$. As with any iterative process, the convergence of the conjugate gradient is halted when the relative residual between two iterations is below some tolerance. In the first plot (Figure 5) the link between $N$ and the error on resolution of the truncated matrix $(9)$ is considered.

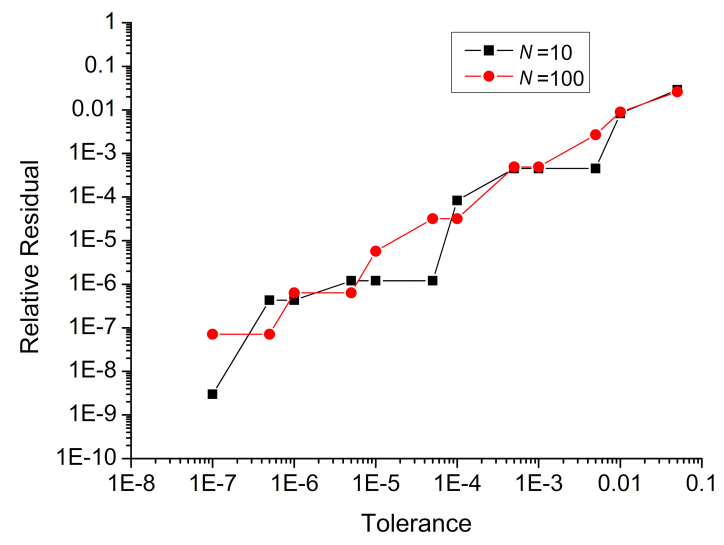

Figure 5: Relative residual values as function of the tolerance, for $N=10$ and $N=100$.

In the second analysis (see Figure 6) the influence of the truncation value $N$ on the number of iterations to solve the problem with the conjugate gradient 
method is considered. The larger $N$, the greater the number of iterations needed to reach tolerance (here fixed to $=10^{-6}$ ). It can be observed that the increase in the number of iterations is quite moderate. Note that the system 12 is close to diagonal, so it is natural (tested and checked) that the preconditionning of the system by its diagonal does not significantly improve the convergence rate.

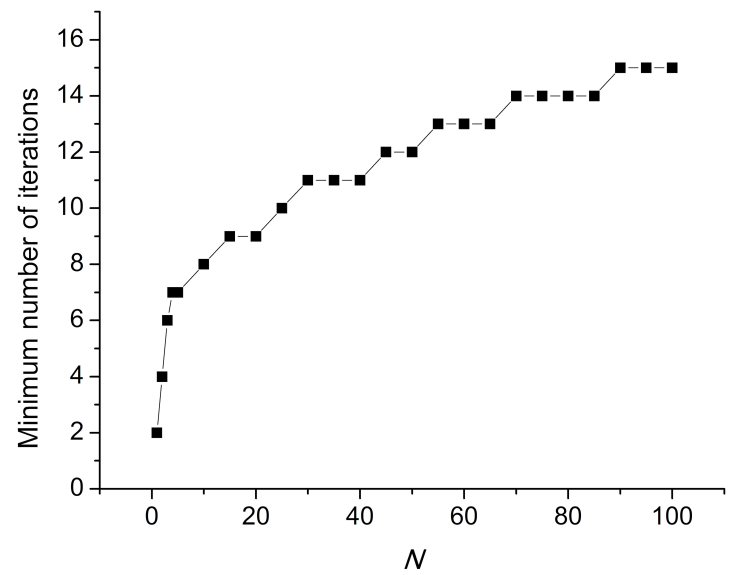

Figure 6: Minimum number of iterations necessary to achieve convergence within the fixed tolerance of Tol $=10^{-6}$, as function of the number of terms $N$ in the multipole expansion.

\section{Appendix B: Scaling analysis}

A change of length-scales is considered in the following way

$$
a_{i} \rightarrow \alpha a_{i}, \quad h \rightarrow \alpha h,
$$

i.e. the multiplication of the length-scales by a factor $\alpha$. By definition, the free charge on each sphere is then given by

$$
\sigma_{f, i} \rightarrow \frac{\sigma_{f, i}}{\alpha^{2}}
$$

so that (6) reveals that

$$
A_{i, \ell} \rightarrow \alpha^{\ell} A_{i, \ell} .
$$

Inserting this scaling of the coefficients in the force, i.e. into (11), reveals the scaling

$$
F \rightarrow \frac{F}{\alpha^{2}}
$$


Further, studying the scaling properties of $\sigma$ via (4) it can be observed the scaling of

$$
\sigma_{i} \rightarrow \frac{\sigma_{i}}{\alpha^{2}}
$$

Now, consider the force as a function of the separation distance $s$ given by $F(s)$. Introducing the scaled variable $\hat{s}=\alpha s$, the force in the scaled situation is given by

$$
\hat{F}(\hat{s})=\hat{F}(\alpha s)=\frac{F(s)}{\alpha^{2}}
$$

and the critical separation distance $s^{\prime}$ where the energy barrier is located is characterised by

$$
F\left(s^{\prime}\right)=0
$$

in the original unscaled situation. Now, it can be concluded that the critical separation distance where the energy barrier is located in the scaled coordinates is given by $\hat{s}^{\prime}=\alpha s^{\prime}$ since

$$
\hat{F}\left(\hat{s}^{\prime}\right)=\hat{F}\left(\alpha s^{\prime}\right)=\frac{F\left(s^{\prime}\right)}{\alpha^{2}}=0 .
$$

Having established the correct scaling for the separation distance (which is of course not a surprise as this is a length scale), scaling of the barrier can be established. Recalling that

$$
E\left(s^{\prime}\right)=-\int_{\infty}^{s^{\prime}} F(s) d s
$$

the energy barrier in the scaled system is

$$
\hat{E}\left(\hat{s}^{\prime}\right)=-\int_{\infty}^{\hat{s}^{\prime}} \hat{F}(\hat{s}) d \hat{s}=-\alpha \int_{\infty}^{s^{\prime}} \hat{F}(\alpha s) d s,
$$

using the change of coordinates $\hat{s}=\alpha s$ in the substitution rule. Applying (13) yields

$$
\hat{E}\left(\hat{s}^{\prime}\right)=-\frac{1}{\alpha} \int_{\infty}^{s^{\prime}} F(s) d s=\frac{1}{\alpha} E\left(s^{\prime}\right) .
$$

These simple considerations reveal the scaling properties in the length scale. A scaling of the charges $q_{i} \rightarrow \alpha q_{i}$ is trivial to understand by looking at the equations. 
A careful examination of Table 1 illustrates indeed that if the radii of the particles are multiplied by a factor $\alpha$, the energy barrier will change by a factor $1 / \alpha$. For instance, the case where $a_{1}=a_{2}=5 \mathrm{~nm}$ and $q_{1}=q_{2}=1$ e has an energy barrier of $0.13 \mathrm{eV}$ and, by doubling the size of each particle, the energy barrier decreases by half (note that the values shown on Table 1 have been rounded to two significant figures and therefore might not reflect with absolute accuracy the results obtained by such rescaling). A further increase in particle radii by a factor of 2 brings a further decrease in the energy barrier to $0.032 \mathrm{eV}$. Such rescaling also occurs with the electrostatic force as described above but by $1 / \alpha^{2}$ as observed elsewhere [28. As revealed by the scaling analysis, all length scales are multiplied by the factor $\alpha=2$, which also applies to the separation distance $s^{\prime}$ where the maximum electrostatic potential energy occurs. This statement has also been confirmed numerically. Further, it is also confirmed numerically that the magnitude of the charge density at each point on the surface of a particle is rescaled by $1 / \alpha^{2}$ (as shown in Figure 7 ), since the surface area is proportional to the radius squared. 

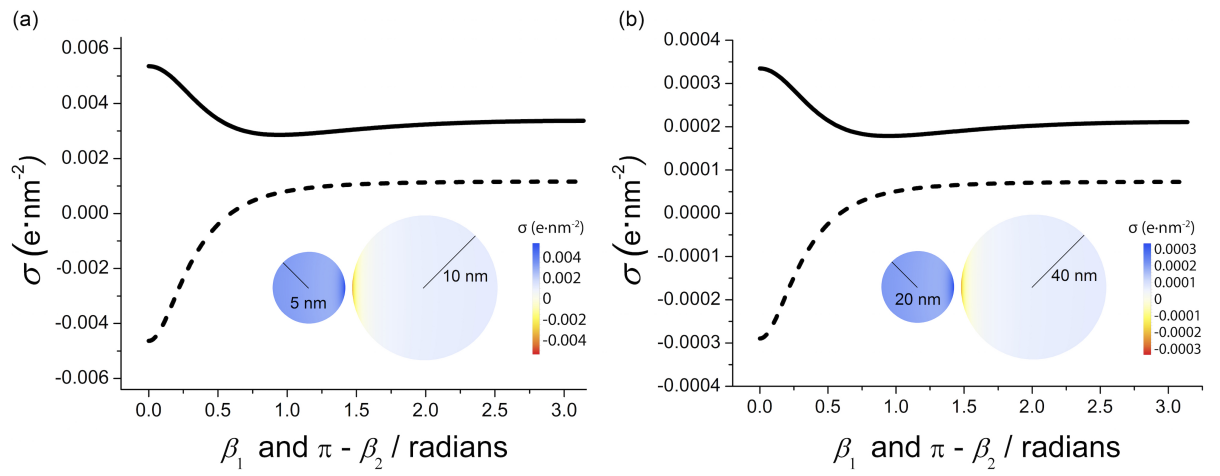

Figure 7: Surface charge density as a function of the polar angle (see Figure 4 calculated for two metastable cases identified in the main text: (a) $a_{1}=5 \mathrm{~nm}, a_{2}=10$ $\mathrm{nm}$, and $s=1 \mathrm{~nm}$; (b) $a_{1}=20 \mathrm{~nm}, a_{2}=40 \mathrm{~nm}$, and $s=4 \mathrm{~nm}$. For the two cases, $k_{1}=k_{2}=20$ and $q_{1}=q_{2}=1$. Note that after a length rescaling by a factor $\alpha=4$, the magnitude of the charge density changes but the charge distribution remains the same. Also shown is the charge density map plotted on the surface of each sphere for each case. 


\section{References}

[1] F. M. Flasar, R. K. Achterberg, B. J. Conrath, P. J. Gierasch, V. G. Kunde, C. A. Nixon, G. L. Bjoraker, D. E. Jennings, P. N. Romani, A. A. SimonMiller, B. Bézard, A. Coustenis, P. G. J. Irwin, N. A. Teanby, J. Brasunas, J. C. Pearl, M. E. Segura, R. C. Carlson, A. Mamoutkine, P. J. Schinder, A. Barucci, R. Courtin, T. Fouchet, D. Gautier, E. Lellouch, A. Marten, R. Prangé, S. Vinatier, D. F. Strobel, S. B. Calcutt, P. L. Read, F. W. Taylor, N. Bowles, R. E. Samuelson, G. S. Orton, L. J. Spilker, T. C. Owen, J. R. Spencer, M. R. Showalter, C. Ferrari, M. M. Abbas, F. Raulin, S. Edgington, P. Ade, E. H. Wishnow, Titan's atmospheric temperatures, winds, and composition, Science 308 (2005) 975-978. doi:10.1126/science.1111150.

[2] S. A. Benner, A. Ricardo, M. A. Carrigan, Is there a common chemical model for life in the universe?, Curr. Opin. Chem. Biol. 8 (2004) 672-689. doi:10.1016/j.cbpa.2004.10.003

[3] C. P. McKay, H. D. Smith, Possibilities for methanogenic life in liquid methane on the surface of Titan, Icarus 178 (2005) 274-276. doi:10. $1016 /$ j.icarus.2005.05.018.

[4] F. Raulin, C. Brassé, O. Poch, P. Coll, Prebiotic-like chemistry on Titan, Chem. Soc. Rev. 41 (2012) 5380-5393. doi:10.1039/C2CS35014A.

[5] J. Stevenson, J. Lunine, P. Clancy, Membrane alternatives in worlds without oxygen: Creation of an azotosome, Sci. Adv. 1 (2015) e1400067. doi:10.1126/sciadv.1400067.

[6] A. J. Coates, F. J. Crary, G. R. Lewis, D. T. Young, J. H. Waite Jr., E. C. Sittler Jr., Discovery of heavy negative ions in Titan's ionosphere, Geophys. Res. Lett. 34 (2007) L22103. doi:10.1029/2007GL030978.

[7] M. L. Cable, S. M. Hörst, R. Hodyss, P. M. Beauchamp, M. A. Smith, P. A. 
[13] P. Lavvas, R. V. Yelle, C. A. Griffith, Titan's vertical aerosol structure at the Huygens landing site: Constraints on particle size, density, charge, Huygens era, Chem. Rev. 112 (2012) 1882-1909. doi:10.1021/cr200221x

[8] P. Lavvas, R. V. Yelle, T. Koskinen, A. Bazin, V. Vuitton, E. Vigren, M. Galand, A. Wellbrocke, A. J. Coates, J.-E. Wahlund, F. J. Crary, D. Snowden, Aerosol growth in Titan's ionosphere, Proc. Natl. Acad. Sci. USA 110 (2013) 2729-2734. doi:10.1073/pnas.1217059110.

[9] J. H. Waite Jr., D. T. Young, T. E. Cravens, A. J. Coates, F. J. Crary, B. Magee, J. Westlake, The process of tholin formation in Titan's upper atmosphere, Science 316 (2007) 870-875. doi:10.1126/science.1139727.

[10] J.-E. Wahlund, M. Galand, I. Müller-Wodarg, J. Cui, R. V. Yelle, F. J. Crary, K. Mandt, B. Magee, J. H. Waite Jr., D. T. Young, A. J. Coates, P. Garnier, K. Ågren, M. André, A. I. Eriksson, T. E. Cravens, V. Vuitton, D. A. Gurnett, W. S. Kurth, On the amount of heavy molecular ions in a Titan's ionosphere, Planet. Space Sci. 57 (2009) 1857-1865. doi:10.1016/ j.pss.2009.07.014

[11] K. Ågren, N. J. T. Edberg, J.-E. Wahlund, Detection of negative ions in the deep ionosphere of Titan during the Cassini T70 flyby, Geophys. Res. Lett. 39 (2012) L10201. doi:10.1029/2012GL051714.

[12] P. Lavvas, R. V. Yelle, V. Vuitton, The detached haze layer in Titan's mesosphere, Icarus 201 (2009) 626-633. doi:10.1016/j.icarus.2009. 01.004

and refractive index, Icarus 210 (2010) 832-842. doi:10.1016/j.icarus. 2010.07 .025

[14] P. Lavvas, M. Sander, M. Kraft, H. Imanaka, Surface chemistry and par-

Willis, Titan tholins: simulating Titan organic chemistry in the Cassiniticle shape: Processes for the evolution of aerosols in Titan's atmosphere, Astrophys. J. 728 (2011) 80. doi:10.1088/0004-637X/728/2/80. 
[15] V. Vuitton, R. V. Yelle, M. J. McEwan, Ion chemistry and N-containing molecules in Titan's upper atmosphere, Icarus 191 (2007) 722-742. doi: $10.1016 / j$. icarus.2007.06.023.

[16] Y. V. Skorov, H. U. Keller, A. V. Rodin, Optical properties of aerosols in Titan's atmosphere, Planet. Space Sci. 56 (2008) 660-668. doi:10.1016/ j.pss.2007.11.013

[17] A. J. Coates, A. Wellbrock, G. R. Lewis, G. H. Jones, D. T. Young, F. J. Crary, J. H. Waite Jr., Heavy negative ions in Titan's ionosphere: Altitude and latitude dependence, Planet. Space Sci. 57 (2009) 1866-1871. doi: $10.1016 / \mathrm{j} \cdot \mathrm{pss} .2009 .05 .009$

[18] V. Vuitton, P. Lavvas, R. V. Yelle, M. Galand, A. Wellbrock, G. R. Lewis, A. J. Coates, J.-E.Wahlund, Negative ion chemistry in Titan's upper at390 \@ mosphere, Planet. Space Sci. 57 (2009) 1558-1572. doi:10.1016/j.pss. 2009.04 .004

[19] H. Imanaka, M. A. Smith, Formation of nitrogenated organic aerosols in the Titan upper atmosphere, Proc. Natl. Acad. Sci. USA 107 (2010) 1242312428. doi:10.1073/pnas.0913353107.

[20] Y. V. Skorov, H. U. Keller, A. V. Rodin, Optical properties of aerosols in Titan's atmosphere: Large fluffy aggregates, Planet. Space Sci. 58 (2010) 1802-1810. doi:10.1016/j.pss.2010.08.002.

[21] E. C. Sittler Jr., A. Ali, J. F. Cooper, R. E. Hartle, R. E. Johnson, A. J. Coates, D. T. Young, Heavy ion formation in Titan's ionosphere: Magnetospheric introduction of free oxygen and a source of Titan's aerosols?, Planet. Space Sci. 57 (2009) 1547-1557. doi:10.1016/j.pss.2009.07.017.

[22] M. Tomasko, R. West, Aerosols in Titan's atmosphere, in: R. H. Brown, J.-P. Lebreton, J. H. Waite (Eds.), Titan from Cassini-Huygens, Springer, 2010, pp. 297-321. doi:10.1007/978-1-4020-9215-2. 
[23] M. Michael, S. N. Tripathi, P. Arya, A. Coates, A. Wellbrock, D. T. Young, High-altitude charged aerosols in the atmosphere of Titan, Planet. Space Sci. 59 (2011) 880-885. doi:10.1016/j.pss.2011.03.010

[24] M. G. Tomasko, L. Doose, S. Engel, L. E. Dafoe, R. West, M. Lemmon, E. Karkoschka, C. See, A model of Titan's aerosols based on measurements 410 made inside the atmosphere, Planet. Space Sci. 56 (2008) 669-707. doi: $10.1016 / j \cdot$.ps .2007 .11 .019

[25] M. G. Tomasko, L. R. Doose, L. E. Dafoe, C. See, Limits on the size of aerosols from measurements of linear polarization in Titan's atmosphere, Icarus 204 (2009) 271-283. doi:10.1016/j.icarus.2009.05.034.

[26] E. Bichoutskaia, A. L. Boatwright, A. Khachatourian, A. J. Stace, Electrostatic analysis of the interactions between charged particles of dielectric materials, J. Chem. Phys. 133 (2010) 024105. doi:10.1063/1.3457157.

[27] C. Wohlfarth, Permittivity (dielectric constant) of liquids, in: W. M. Haynes (Ed.), CRC Handbook of Chemistry and Physics (Internet edition),

[28] E. B. Lindgren, H.-K. Chan, A. J. Stace, E. Besley, Progress in the theory of electrostatic interactions between charged particles, Phys. Chem. Chem. Phys. 18 (2016) 5883-5895. doi:10.1039/C5CP07709E

[29] D. Snowden, R. V. Yelle, J. Cui, J.-E. Wahlund, N. J. T. Edberg, K. Ågren, The thermal structure of Titan's upper atmosphere, I: Temperature profiles

from Cassini INMS observations, Icarus 226 (2013) 552-582. doi : 10.1016/ j.icarus.2013.06.006.

[30] B. A. Tinsley, R. P. Rohrbaugh, M. Hei, K. V. Beard, Effects of image charges on the scavenging of aerosol particles by cloud droplets and on ${ }_{430}$ droplet charging and possible ice nucleation processes, J. Atmos. Sci. 57

口) (2000) 2118-2134. doi:10.1175/1520-0469(2000)057<2118:EOICOT>2. $0 . \mathrm{CO} ; 2$. 
[31] K. S. Carslaw, R. G. Harrison, J. Kirkby, Cosmic rays, clouds, and climate, Science 298 (2002) 1732-1737. doi:10.1126/science.1076964.

[32] S. N. Tripathi, R. G. Harrison, Enhancement of contact nucleation by scavenging of charged aerosol particles, Atmos. Res. 62 (2002) 57-70. doi:10.1016/S0169-8095(02)00020-0.

[33] A. J. Coates, A. Wellbrock, G. R. Lewis, G. H. Jones, D. T. Young, F. J. Crary, J. H. Waite, R. E. Johnson, T. W. Hill, E. C. Sittler Jr., Negative ions at Titan and Enceladus: recent results, Farad. Discuss. 147 (2010) 293-305. doi:10.1039/c004700g.

[34] O. Shebanits, J.-E. Wahlund, K. Mandt, K. Ågren, N. J. T. Edberg, J. H. Waite Jr., Negative ion densities in the ionosphere of Titan Cassini RPWS/LP results, Planet. Space Sci. 84 (2013) 153-162. doi: $10.1016 / j \cdot p s s .2013 .05 .021$

[35] A. Wellbrock, A. J. Coates, G. H. Jones, G. R. Lewis, J. H. Waite, Cassini CAPS-ELS observations of negative ions in Titan's ionosphere: Trends

a of density with altitude, Geophys. Res. Lett. 40 (2013) 4481-4485. doi: $10.1002 / \mathrm{grl} .50751$

[36] P. Wang, A. Anderko, Computation of dielectric constants of solvent mixtures and electrolyte solutions, Fluid Phase Equilib. 186 (2001) 103-122. doi:10.1016/S0378-3812(01)00507-6.

[37] A. Khain, V. Arkhipov, M. Pinsky, Y. Feldman, Y. Ryabov, Rain enhancement and fog elimination by seeding with charged droplets. Part I: Theory and numerical simulations, J. Appl. Meteorol. 43 (2004) 1513-1529. doi:10.1175/JAM2131.1. 